megalencephaly. L Pediatr August 1997;131:320-324). (Reprints: Adrian D Sandler MD, Center for Child Development, Thoms Rehabilitation Hospital, PO Box 15025, Asheville, NC 28813).

COMMENT. Idiopathic megalencephaly in school-age children, sometimes considered 'benign.' may be associated with subtle motor impairments and neurodevelopmental dysfunction.

\title{
INFANT CT-DILATED VENTRICLES AND LEARNING DISORDERS
}

The risk of developing learning disability at school age in extremely low birth weight (ELBW) infants showing dilated lateral ventricles on CT at postconceptual age of 40 weeks was evaluated at Akita School of Medicine, Japan. The mean area of lateral ventricles, measured by computer digitizer, was significantly larger in the learning disability group of 20 children than controls. None had progressive hydrocephalus, and the dilated ventricles were secondary to brain atrophy. (Ishida A, Nakajima W, Arai H et al. Cranial computed tomography scans of premature babies predict their eventual learning disabilities. Pediatr Neurol May 1997;16:319-322). (Respond: Dr Ishida, Department of Pediatrics, Akita University School of Medicine, 1-1-1 Hondo, Akita-shi, Akita 010, Japan).

COMMENT. Measurement of lateral ventricles by cranial CT at corrected term in ELBW newborns is an early predictor of learning disabilities at school age, and provides an opportunity for early educational intervention.

ADHD in low birth weight children. Neonatal cranial ultrasound abnormalities suggestive of white matter injury in low-birth-weight children were an increased risk for neuropsychiatric disorders by age 6 years in a study at Columbia University and New York State Psychiatric Institute. (Whitaker AH, Van Rossem R, Feldman JF et al. Psychiatric outcomes in lowbirth-weight children at age 6 years: Relation to neonatal cranial ultrasound abnormalities. Arch Gen Psychiatry Sept 1997;54:847-856). Twenty-two percent of the cohort were affected; ADHD was the most common disorder (16\%).

\section{DIET AND INFANT BEHAVIOR}

The relation between rate of weight gain and diet-dependent changes in biochemistry, physiology and behavior of 142 preterm infants (mean birthweight $1364 \mathrm{~g}$ ) fed varied protein and energy intakes was evaluated at the College of Physicians and Surgeons, Columbia University, New York. Rapidly growing infants had increased heart rates, respiratory rates, active sleep time, and decreased spectral edge EEG frequencies compared to slow growers. The changes in autonomic responses related to diet and rapid growth were explained by an hypothesis of shifts in the balance of catecholamine and serotonergic neurotransmitter systems. (de Klerk A, Schulze KF, Kashyap S, Sahni R, Fifer W, Myers M. Diet and infant behavior. Acta Paediatr Suppl 422 July 1997;86:65-68). (Respond: Dr KF Schulze, Department of Pediatrics, College of Physicians and Surgeons, Columbia University, 630 West 168th St, New York, NY 10032).

COMMENT. This study of diet and behavior in LBW infants was stimulated by reports of the influence of infant diets on later adult morbidity. The authors were particularly interested in early diet in relation to adult diseases such as hypertension. The findings might also apply to nervous system development and a possible diet related mechanism of attention deficit and 
learning disorders. The incidence of subsequent ADHD among these infants would be of interest.

\section{DYSMORPHIC SYNDROMES AND LEARNING DISABILITIES}

Four cases of Kabuki make-up syndrome (KMS) from various ethnic groups in Vancouver, British Columbia, are reported from the Sunny Hill Health Centre for Children, Vancouver. Three were diagnosed late, at 7 years or older. The cardinal manifestations are: 1) dysmorphic facies - long palpebral fissures, large ears, arched eyebrows, outer one third of the lower eyelid turned outward, similar to make-up of Kabuki actors in Japanese theatre; 2) brachydactyly of 5th finger; 3) increased digital ulnar loops; 4) mental retardation; 5) short stature. All 4 patients presented early with delayed speech, all had autistic-like behavior, and 2 had repetitive stereotyped behavior. All showed variable degrees of learning disabilities and/or retardation, and 2 had declines in IQ in adolescence. (Ho HH, Eaves LC. Kabuki make-up (Niikawa-Kuroki) syndrome: cognitive abilities and autistic features. Dev Med Child Neurol July 1997;39:487-490). (Respond: Helena H Ho, MD FRCPC, Sunny Hill Health Centre for Children, 3644 Slocan St, Vancouver, BC, Canada V5M 3E8).

COMMENT. The diagnosis of Kabuki make-up syndrome should be considered in dysmorphic children with microcephaly and presenting with learning disabilities and/or autism. Although the majority of cases are Asian from Japan, KMS occurs in other Asians, Europeans, South American, Arab, Indian, and Chinese. The condition may be overlooked in Asians outside Japan, since the long palpebral fissures and short stature can be accepted as normal.

\section{OBSESSIVE-COMPULSIVE DISORDER}

\section{STRIATAL MRI ABNORMALITIES IN OCD}

MRI scans from 19 children aged 7 to 18 years, with recent onset obsessive-compulsive disorder (OCD), untreated with psychotropic drugs, and 19 matched controls, were analyzed for striatal volume at Western Psychiatric Institute, University of Pittsburgh, PA. Patients with OCD had significantly smaller putamen and total striatal volumes ( $7 \%$ reduction) and larger third ventricles than controls. Prefrontal cortical, white matter, lateral ventricular, and intracranial volumes were not altered. Reduced striatal volume was inversely correlated with severity of OCD symptoms, but not with illness duration or age of onset. Males and females were equally involved. (Rosenberg DR, Keshavan MS, O'Hearn KM et al. Frontostriatal measurement in treatmentnaive children with obsessive-compulsive disorder. Arch Gen Psychiatry Sept 1997;54:824-830). (Reprints: David R Rosenberg MD, Wayne State University School of Medicine, Psychiatry 9B, 4201 St Antoine Blvd, Detroit, MI 48201).

COMMENT. This study confirms the striatal pathology in children with obsessive-compulsive disorder, and emphasizes the role of the putamen in OCD. The findings were not related to prior medication effects or comorbid illness such as depression or anxiety.

Oculomotor response inhibition abnormalities in OCD are reported by the above authors in a study of 18 untreated children, aged 8 to 16 years, compared to 18 controls. The ability to suppress behavioral responses related to prefrontal cortical function was reduced in OCD patients. (Rosenberg DR, Averbach DH, O'Hearn KM et al. Arch Gen Psychiatry Sept 1997;54:831-838). 